\title{
Kopplung eines auf der Momentenmethode basierenden Computerprogramms mit einem FEM-Algorithmus zur Berechnung von elektromagnetischen Streuproblemen im medizinischen Bereich
}

\author{
M. Schick and F. M. Landstorfer \\ Universität Stuttgart, Institut für Hochfrequenztechnik, Pfaffenwaldring 47, 70550 Stuttgart, Germany
}

\begin{abstract}
Zusammenfassung. Am Beispiel der Verkopplung von medizinischen Geräten über den menschlichen Körper werden elektromagnetische Störphänomene im Klinikbereich betrachtet. Für die Berechnung dieser komplexen Szenarien wird zum einen die Momentenmethode (MoM) verwendet, die sich in besonderem Maße für die Berücksichtigung metallischer Strukturen und offener Streuprobleme eignet, und zum anderen die Methode der Finiten Elemente (FEM), mit der die Eigenschaften des menschlichen Körpers besser berücksichtigt werden können. Mit Hilfe des Äquivalenzprinzips lässt sich das Gesamtproblem in zwei Teile zerlegen, in ein inneres und in ein äußeres. Der Außenraum wird dabei mit der MoM behandelt und das Innere, d.h. der Körper mit der FEM. Die Kopplung der beiden Methoden erfolgt an der Körperoberfläche über äquivalente Oberflächenströme. Durch Lösen des resultierenden linearen Gleichungssystems für das gesamte Problem lassen sich dann die Oberflächenströme und die über die Kontinuitätsgleichung miteinander verknüpften elektromagnetischen Felder bestimmen.
\end{abstract}

\section{Einleitung}

Die zu simulierenden Szenarien der Verkopplung von Medizingeräten über den menschlichen Körper sind sehr komplex, da der Einfluss des Patienten auf die direkte Verkopplung, zum Beispiel über Gewebeströme, entscheidende Bedeutung hat. Aus diesem Grunde müssen neue Hybridverfahren entwickelt werden. Die Momentenmethode (MoM) eignet sich besonders für offene Streuprobleme, da zum einen lediglich Gebiete, in denen Ladungen und Ströme auftreten, diskretisiert werden müssen, und zum anderen die Unendlichkeit des freien Raumes durch eine Greensche Funktion exakt nachgebildet wird. Der Vorteil der Methode der Finiten Elemente ist der sehr hohe Freiheitsgrad bei der Zerlegung des Lösungsraums in Finite Elemente, weshalb auch komplizierte Geo-

Correspondence to: M. Schick

(schick@ihf.uni-stuttgart.de) metrien und Inhomogenitäten gut modelliert werden können. Diese Vorteile der beiden Methoden werden hier ausgenützt indem sie zu einem hybriden Verfahren kombiniert werden.

\section{2 Äquivalenzprinzip}

In Abb. 1 ist ein Beispiel für ein allgemeines Streuproblem dargerstellt. Ein dielektrischer Streukörper mit den Materialparametern $\mu_{r} \mu_{0}$ und $\varepsilon_{r} \varepsilon_{0}$ befindet sich im unendlich ausgedehnten Freiraum $\left(\mu_{0}\right.$ und $\left.\varepsilon_{0}\right)$. Allgemein ist auch die Verwendung von inhomogenen Dielektrika denkbar. Die Anregung erfolgt beispielsweise durch eine einfallende ebene Welle, so dass außerhalb des dielektrischen Körpers ein elektromagnetisches Feld $\boldsymbol{E}_{\text {ext }}$ und $\boldsymbol{H}_{\text {ext }}$ vorherrscht und im Inneren ein Feld $\boldsymbol{E}_{\text {int }}$ und $\boldsymbol{H}_{\text {int }}$ (vgl. Harrington, 1968 und Jakobus, 1995).

Dieses allgemeine elektromagnetische Streuproblem lässt sich nun mit Hilfe des Äquivalenzprinzips in ein externes äquivalentes und ein internes äquivalentes Problem überführen. Beim externen Äquivalent (siehe Abb. 2 links) werden die Felder im Außenraum betrachtet und die Wirkung der Felder im Inneren des dielektrischen Körpers durch äquivalente elektrische und magnetische Oberflächenströme

$\boldsymbol{J}_{S}=\hat{n} \times \boldsymbol{H}(O)$ und $\boldsymbol{M}_{S}=-\hat{n} \times \boldsymbol{E}(O)$

auf der Grenzschicht des Körpers beschrieben. Damit kann das Innere als feldfrei angenommen werden und somit auch mit einem beliebigen Material gefüllt werden (also z.B. auch mit $\mu_{0}$ und $\varepsilon_{0}$ ). Das interne Äquivalent (siehe Abb. 2 rechts) stellt den umgekehrten Fall dar. Hierbei werden die Felder im Innenraum des dielektrischen Körpers betrachtet und die Wirkung der Felder im Außenraum durch äquivalente Oberflächenströme beschrieben. Hier kann das Äußere als feldfrei angenommen werden und somit auch mit einem beliebigen Material gefüllt werden (also z.B. auch mit $\mu_{r} \mu_{0}$ und $\varepsilon_{r} \varepsilon_{0}$ ).

Die äquivalenten Oberflächenströme für den externen und den internen Fall müssen identisch sein, aufgrund der notwendigen Stetigkeit der Tangentialenkomponenten der 


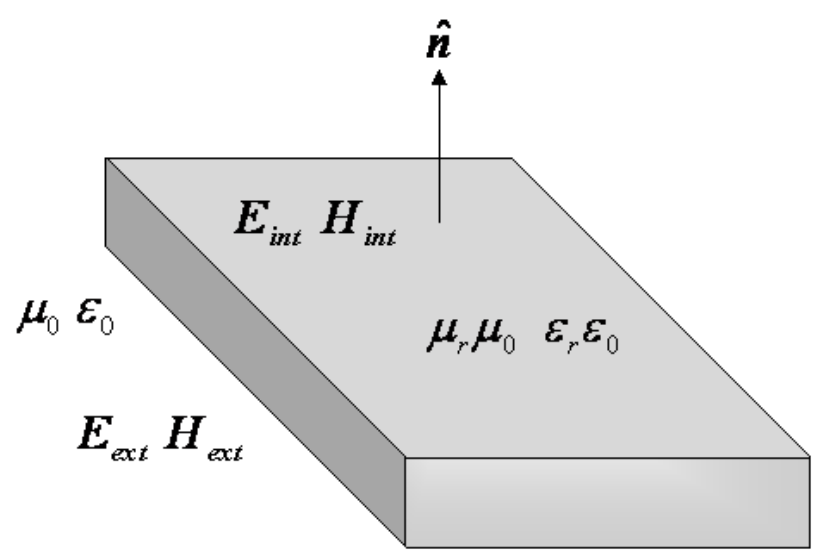

Abbildung 1. Allgemeines elektromagnetisches Streuproblem.

elektrischen und magnetischen Felder an der Oberfläche des Dielektrikums beim ursprünglichen Problem. Nach dem Äquivalenzprinzip können also die Felder an der Grenzfläche über die Oberflächenströme miteinander verkoppelt werden.

\section{Verwendete Methoden}

Bei der Momentenmethode handelt es sich um ein Integralgleichungsverfahren. Sie ist besonders für metallische Strukturen und offene Streuprobleme geeignet. Die elektrische Feldstärke lässt sich in Abhängigkeit von Potenzialen formulieren, welche sich ihrerseits in Abhängigkeit von elektrischen und magnetischen Oberflächeströmen ausdrücken lassen. Zur Bestimmung dieser Ströme erfolgt eine Diskretisierung der Oberflächen in dreieckförmige Flächenelemente. Auf den Kanten der Dreiecke werden die Oberflächenströme als gewichtete Summe von Basisfunktionen $\boldsymbol{f}_{n}$ nach Rao et al. (1982) angesetzt.

$\boldsymbol{J}_{S}=\sum_{n=1}^{N_{J}} \alpha_{J} \cdot \boldsymbol{f}_{J, n}$ und $\boldsymbol{M}_{S}=\sum_{n=1}^{N_{M}} \alpha_{M} \cdot \boldsymbol{f}_{M, n}$.

Es erfolgt eine Gewichtung nach dem Galerkinverfahren mit denselben Funktionen $\boldsymbol{f}_{n}$. Im MoM-Gebiet resultiert dann das folgende lineare Gleichungssystem mit den unbekannten Oberflächenströmen $J$ und elektrischen Feldern $E_{d}$ auf der Oberfläche des Dielektrikums, sowie einer einfallenden anregenden Feldstärke $E^{\text {inc: }}$

$$
\left[\begin{array}{ll}
C_{d d} & C_{d l} \\
C_{l d} & C_{l l}
\end{array}\right] \cdot \underbrace{\left\{\begin{array}{c}
J_{d} \\
J_{l}
\end{array}\right\}}_{\text {unbekannt }}=\left[\begin{array}{cc}
D_{d d} & 0 \\
D_{l d} & 0
\end{array}\right] \cdot \underbrace{\left\{\begin{array}{c}
E_{d} \\
0
\end{array}\right\}}_{\text {unbekannt }}-\underbrace{\left\{\begin{array}{c}
E_{d}^{\text {inc }} \\
E_{l}^{\text {inc }}
\end{array}\right\}}_{\text {Anregung }}
$$

Bei der Methode der Finiten Elemente handelt es sich um ein partielles Differenzialgleichungsverfahren. Ausgehend von den ersten beiden Maxwellschen Gleichungen erhält man durch Bildung der Rotation und in einander Einsetzen die Wellengleichungen für die elektrische bzw. magnetische Feldstärke. Nun erfolgt zuerst eine Gewichtung mit den Formfunktionen $\boldsymbol{w}_{n}$ (Kantenelemente 1. Ordnung) nach Jin

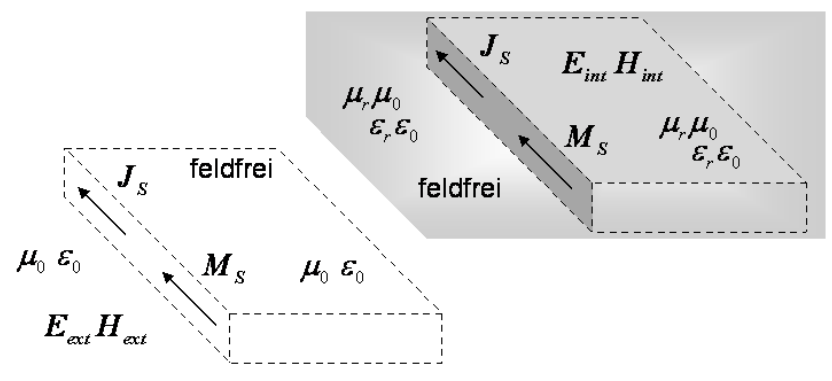

Abbildung 2. Externes (links) und internes (rechts) äquivalentes Problem.

(1993) bevor dann die Diskretisierung des zu behandelnden Volumens in Tetraeder vorgenommen wird. Die elektrische Feldstärke wird hierbei als Überlagerung von Basisfunktionen angesetzt:

$\boldsymbol{E}(\boldsymbol{r})=\sum_{n=1}^{N_{E}} E_{n} \cdot \boldsymbol{w}_{n}(\boldsymbol{r})$.

Als resultierendes lineares Gleichungssystem für den FEMBereich ergibt sich damit dann:

$$
\left[\begin{array}{cc}
A_{i i} & A_{i d} \\
A_{d i} & A_{d d}
\end{array}\right] \cdot \underbrace{\left\{\begin{array}{c}
E_{i} \\
E_{d}
\end{array}\right\}}_{\text {unbekannt }}=\left[\begin{array}{cc}
0 & 0 \\
0 & B_{d d}
\end{array}\right] \cdot \underbrace{\left\{\begin{array}{c}
0 \\
J_{d}
\end{array}\right\}}_{\text {unbekannt }}+\underbrace{\left\{\begin{array}{c}
G^{i n t} \\
0
\end{array}\right\}}_{\text {Anregung }}
$$

mit den unbekannten elektrischen Feldern im Dielektrikum $E_{i}$ und auf dessen Oberfläche $E_{d}$, sowie den Oberflächenströmen $J_{d}$ und einer möglichen Anregung im Inneren des dielektrischen Körpers $G^{\text {int }}$.

\section{Kopplung von MoM und FEM}

Bisher wurden das externe und das interne Äquivalent unabhängig von einander betrachtet und für beide Teilprobleme jeweils ein lineares Gleichungssystem hergeleitet, dass einen Zusammenhang zwischen den unbekannten komplexen Koeffizienten $J$ und $E$ beschreibt. Nun erfolgt die Kopplung der beiden Verfahren nach dem oben beschriebenen Äquivalenzprinzip. Dabei sind die Basisfunktionen auf der Oberfläche mit den Formfunktionen im Inneren des dielektrischen Körpers an der Oberfläche über die Beziehung

$\boldsymbol{w}_{n}(\boldsymbol{r})=\hat{n} \times\left.\boldsymbol{f}_{n}\right|_{S}$

mit einander verknüpft. Formell erfolgt die Kopplung über die Oberflächenströme $\boldsymbol{J}$. Es wird Gleichung (3) nach $\boldsymbol{J}$ aufgelöst und in Gleichung (5) eingesetzt. Das so entstehende Gleichungssystem lässt sich in das folgende überführen:

$\left[\begin{array}{ll}A_{i i} & A_{i d} \\ A_{d i} & A_{d d}^{\prime}\end{array}\right] \cdot \underbrace{\left\{\begin{array}{c}E_{i} \\ E_{d}\end{array}\right\}}_{\text {unbekannt }}=\underbrace{\left\{\begin{array}{c}0 \\ \left.E^{s r c}\right\}\end{array}\right\}+\left\{\begin{array}{c}G^{i n t} \\ 0\end{array}\right\}}_{\text {Anregung }}$.

Dieses wird dann mit dem Konjugierten-GradientenVerfahren iterativ gelöst. 

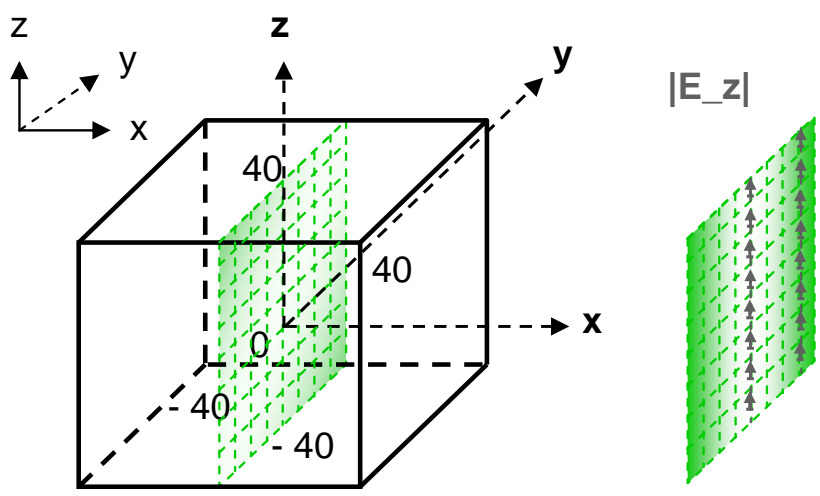

Abbildung 3. Dielektrischer Würfel mit einer Berechnungsebene für die elektrischen Feldstärken.

Zur Validierung des hybriden Verfahrens wird eine Vergleichsrechnung mit der Momentenmethode bei einem homogenen dielektrischen Würfel vorgenommen (siehe Abb. 3). Als Anregung dient eine einfallende ebene Welle.

Betrachtet wird das resultierende elektrische Nah- und Fernfeld (siehe Abb. 4). Es ergibt sich eine gute Übereinstimmung der Ergebnisse beider Verfahren, so dass mit dem hier beschriebenen hybriden Ansatz jetzt weitere Untersuchungen angestrebt werden.

\section{Zusammenfassung}

Es wird die Kopplung der Momentenmethode mit der Methode der Finiten Elemente beschrieben. Zuerst wird auf das Äquivalenzprinzip eingegangen, nachdem das ursprüngliche Streuproblem in zwei Unterprobleme aufgeteilt werden kann und dass die Grundlage für die Kopplung bildet. Anschließend werden die beiden verwendeten Methoden näher beschrieben. Die Kopplung wird dann über die Oberflächenströme durchgeführt und das resultierende Gleichungssystem gelöst. Eine Validierungsrechnung weist eine gute Übereinstimmung des hybriden Verfahrens im Vergleich zur Lösung mit der Momentenmethode auf.

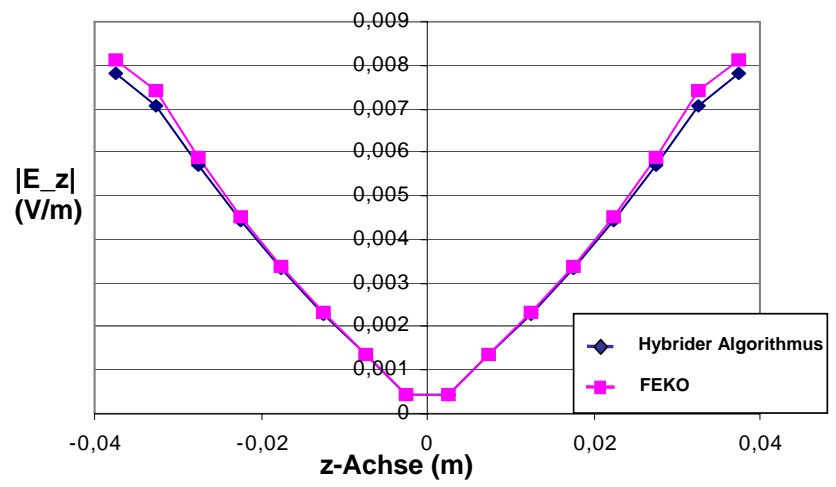

Abbildung 4. Vergleich der berechneten elektrischen Feldstärken.

Danksagung. Die Autoren danken der Deutschen Forschungsgemeinschaft für die Finanzierung des Projektes.

\section{Literatur}

Harrington, R. F.: Field Computation by Moment Methods, Macmillan, New York, 1968.

Jakobus, U.: Erweiterte Momentenmethode zur Behandlung kompliziert aufgebauter und elektrische großer elektromagnetischer Streuprobleme, Nr. 171 in Fortschrittsberichte, Reihe 21, VDI Verlag, Düsseldorf, Dissertationsschrift, 1995.

Jin, J.: The Finite Element Method in Electromagnetics, John Wiley \& Sons, New York, 1993.

Rao, S. M., Wilson, D. R., and Glisson, A. W.: Electromagnetic scattering by surfaces of arbitrary shape, IEEE Transactions on Antennas and Propagation, 30, 3, 409-418, 1982. 